\title{
GI Bleeding Risk of DOACs Versus Warfarin: Is Newer Better?
}

\author{
Linda A. Feagins ${ }^{1,2} \cdot$ Rick A. Weideman ${ }^{3}$ \\ Published online: 18 April 2018 \\ (c) Springer Science+Business Media, LLC, part of Springer Nature 2018
}

Until recently, warfarin had been the mainstay of anticoagulant treatment for patients with atrial fibrillation. With the approval in 2010 of the first direct-acting non-vitamin K oral anticoagulant (DOAC) dabigatran, the choice of therapies has been rapidly evolving. Recent studies reveal that currently half of the patients receiving anticoagulation for atrial fibrillation are taking DOACs as opposed to warfarin [1]. The reason for the rapid acceptance for these drugs has been the convenience of fixed dosing with no need for laboratory monitoring or dietary discretion, as required with warfarin. Despite these apparent advantages, debate has remained regarding the risk of gastrointestinal (GI) bleeding with these agents as compared to warfarin.

The landmark trials that led to the United States Food and Drug Administration (FDA) approval of the currently approved DOACs, including dabigatran, rivaroxaban, apixaban, and edoxaban, reported that the all-cause bleeding rates were similar if not lower with DOACs as compared with warfarin. Nevertheless, the rate of GI bleeding was higher for patients treated with dabigatran [2] (3 vs $2 \%$ ), rivaroxaban [3] (3.2 vs 2.2\%), or edoxaban [4] (1.5 vs $1.2 \%$ ) as compared with patients taking warfarin. On the other hand, the rates of GI bleeding were similar if not lower for patients treated with apixaban [5] as compared to warfarin (1.2 vs 1.3\%). Likewise, an FDA postmarketing study also found the rate of GI bleeding with dabigatran to be higher as compared to warfarin (1.6 versus 1.1 per 100 patient years) [6].

Of the DOACs, dabigatran is the only direct thrombin inhibitor, whereas the others are direct factor Xa inhibitors. While the reason for the higher risk of GI bleeding with

Linda A. Feagins

Linda.Feagins@UTSouthwestern.edu

1 Division of Gastroenterology and Hepatology, VA North Texas Healthcare System, 4500 S. Lancaster Rd 111B1, Dallas, TX 75216, USA

2 Division of Gastroenterology and Hepatology, UT Southwestern Medical Center, Dallas, TX, USA

3 Department of Pharmacy, VA North Texas Healthcare System, Dallas, TX, USA dabigatran is unclear, a few hypotheses exist. Dabigatran is absorbed principally in the stomach and proximal small bowel as an inactive prodrug that is then metabolized to the active drug by serum and hepatic esterases. Nonetheless, the bioavailability of the drug is low (approximately 3-7\%) with the unabsorbed dabigatran being converted to active dabigatran in the distal bowel and then excreted in the feces. This active drug in the distal bowel may promote GI bleeding more than warfarin, which is not activated in the bowel [7]. Secondly, there are reports that dabigatran is associated with esophagitis and gastric ulceration, suggesting the drug may directly injure the gastrointestinal mucosa.

In this issue of Digestive Diseases and Sciences, Kolb et al. [8] report a post hoc analysis of the Randomized Evaluation of Long-Term Anticoagulation Therapy (RE-LY) trial (one of the landmark trials discussed above, this one comparing dabigatran to warfarin) where they re-reviewed the cases of suspected GI bleeding, collecting additional data including the causative lesions and the site and acuity of bleeding within the bowel. They could localize bleeding in approximately two-thirds of the cases, with $47 \%$ of those cases detected in the upper GI tract and 39\% in the colon. Interestingly, the rate of bleeding in the upper GI tract was similar between the two doses of dabigatran studied (110 and $150 \mathrm{mg}$ ) as compared with warfarin, whereas lower GI bleeding (which included colonic, jejunal, and ileal sources) was more frequent in patients treated with dabigatran as compared with warfarin (relative risk [RR] 1.78 for dabigatran $110 \mathrm{mg}$ and RR 2.23 for dabigatran $150 \mathrm{mg}$ ). The activation of dabigatran (while warfarin remains inactive) in the lower GI tract as described above may explain the higher rates of GI bleeding, especially from the lower GI tract lesions.

The most common culprit lesions identified were gastroduodenal ulcers or erosions $(20 \%)$, colonic diverticula (9\%), occult malignancy (8\%), and angioectasias (6\%). While the authors report that there was no baseline difference in the use of proton pump inhibitors or antiplatelet agents, no data support whether these agents (or even $H$. pylori infection) are associated with the development of 
gastroduodenal ulcers or whether the ulceration is related to an ulcerogenic property of the dabigatran itself. The authors report that angioectasias in the upper GI tract bleed with equal frequency in dabigatran- and warfarin-treated patients, whereas in the colon the lesions only bleed in patients treated with dabigatran. If this finding was truly a consequence of active dabigatran in the colon, one would predict that all rather than a single type of colonic lesion would bleed in the presence of dabigatran. For instance, the rate of bleeding from diverticula, presumably all located in the colon, is the same between those treated with dabigatran and warfarin. Therefore, either there is something different about an angioectasia that increases its propensity to bleed with active dabigatran present that is not true with other lesions, or there is some other explanation, such as a type I error given the relatively small numbers of each type of bleeding lesion in the study.

The severity of GI bleeding was assessed in aggregate and categorized as either "major" $(\geq 2 \mathrm{~g} / \mathrm{dL}$ hemoglobin drop and/or $\geq 2 \mathrm{U}$ red blood cell transfusion) or "life-threatening" ( $\geq 5 \mathrm{~g} / \mathrm{dL}$ hemoglobin drop, $\geq 4 \mathrm{U}$ red blood cell transfusion, inotropic support requirement, need for surgery, and/ or death). In this analysis, there was more life-threatening bleeding, largely accounted for by bleeding from the lower GI tract, in patients treated with dabigatran as compared to warfarin (RR 2.0 for dabigatran $110 \mathrm{mg}$ and RR 2.64 for dabigatran $150 \mathrm{mg}$ versus warfarin). Interestingly, two recent retrospective studies have suggested that the consequences of GI bleeding while receiving a DOAC may be less severe than those who experience GI bleeding while taking warfarin $[9,10]$. Both studies, which included not only dabigatran but also apixaban and rivaroxaban, found that patients treated with a DOAC were less likely to need a blood transfusion and stayed in the hospital fewer days than those who received warfarin. Interestingly, these "realworld" studies also found that the rates of GI bleeding were lower for patients treated with a DOAC than with warfarin. A piece of missing data from the RE-LY analysis is the international normalized ratios (INRs) at presentation of patients who bled while taking warfarin. Both the aforementioned retrospective studies reported average INRs $>3$, and one study reported half of the patients had a supratherapeutic INR on presentation. It is very likely that the patients treated with warfarin in the setting of a randomized trial were much more optimally controlled than the "real-world" patients evaluated in the retrospective studies and may have contributed to apparent lower bleeding rates for those taking warfarin.

The study by Kolb et al. has several strengths, including the large randomized nature of the original study and the large number overall of GI bleeding events. Yet, it is important to keep in mind that the study is less generalizable to all patients treated with anticoagulants, as the authors included only those with non-valvular atrial fibrillation and excluded patients with a history of GI bleeding or peptic ulcer disease. Moreover, as this was a post hoc analysis, GI bleeding was not a primary endpoint in the original study; furthermore, the contribution of concomitant antiplatelet drugs and proton pump inhibitors could not be definitely determined. Lastly, though not analyzed by causes of bleeding, higher blood concentrations of dabigatran are correlated with a higher risk of major bleeding [11]. Since plasma concentrations were unfortunately not included in the Kolb et al. analysis, it is therefore unclear how these levels may affect the risk of GI bleeding in particular. Interestingly, of the data that have been published with regard to plasma concentrations, one of the most significant factors affecting these levels was age that was likely driven by reduced renal function. These older patients were noted to have not only higher rates of major bleeding but also a higher stroke risk. Similarly, a retrospective analysis of dabigatran, rivaroxaban, and warfarin using commercial and Medicare claims data found an increased risk of GI bleeding for both dabigatran and rivaroxaban as compared to warfarin once patients were over the age of 75 [12].

Overall, DOACs have proven promising in regard to safety from dietary and drug interactions when compared to warfarin; the increased risk of GI bleeding can likely be managed by careful patient selection and by checking at least one blood level after starting DOACs, if available. In the case of dabigatran, ensuring that a target range has been met has been suggested in a modeling study to avoid perhaps $20 \%$ of bleeding events [13]. Given current knowledge, where should future studies focus their efforts? Ideally, a randomized controlled trial of patients designed with GI bleeding as a primary outcome, with stratification for concomitant antiplatelet drugs and proton pump inhibitors, should provide useful data in this regard. It would be helpful to include a blood level of dabigatran either near the study start and/or at the time of a bleeding event in order to assess the utility of such a level in determining bleeding risk. For now, patients should be counseled about the pros and cons of the available anticoagulants and about the increased risk of GI bleeding for those taking dabigatran. Lastly, until blood levels of dabigatran are available in the USA, physicians may want to consider apixaban over the other DOACs for patients with a history of GI bleeding or peptic ulcer disease, given higher rates of GI bleeding have not been reported with apixaban.

\section{Compliance with ethical standards}

Conflict of interest The authors declare that they have no conflict of interest. 


\section{References}

1. Barnes GD, Lucas E, Alexander GC, Goldberger ZD. National trends in ambulatory oral anticoagulant use. Am J Med. 2015;128:1300-1305e1302.

2. Connolly SJ, Ezekowitz MD, Yusuf S, et al. Dabigatran versus warfarin in patients with atrial fibrillation. $N$ Engl J Med. 2009;361:1139-1151.

3. Patel MR, Mahaffey KW, Garg J, et al. Rivaroxaban versus warfarin in nonvalvular atrial fibrillation. $N$ Engl J Med. 2011;365:883-891.

4. Giugliano RP, Ruff CT, Braunwald E, et al. Edoxaban versus warfarin in patients with atrial fibrillation. $N$ Engl J Med. 2013;369:2093-2104.

5. Granger CB, Alexander JH, McMurray JJV, et al. Apixaban versus warfarin in patients with atrial fibrillation. $N$ Engl J Med. 2011;365:981-992.

6. Southworth MR, Reichman ME, Unger EF. Dabigatran and postmarketing reports of bleeding. $N$ Engl J Med. 2013;368:1272-1274.

7. Feagins LA. Management of anticoagulants and antiplatelet agents during colonoscopy. Am J Med. 2017;130:786-795.
8. Kolb JM, Flack KF, Chatterjee-Murphy P, et al. The locations and mucosal lesions responsible for major gastrointestinal bleeding in patients on warfarin or dabigatran. Dig Dis Sci. (Epub ahead of print). https://doi.org/10.1007/s10620-018-5007-6.

9. Brodie MM, Newman JC, Smith T, Rockey DC. Severity of gastrointestinal bleeding in patients treated with direct-acting oral anticoagulants. Am J Med. 2017. https://doi.org/10.1016/j.amjme d.2017.11.007.

10. Cangemi DJ, Krill T, Weideman R, Cipher DJ, Spechler SJ, Feagins LA. A comparison of the rate of gastrointestinal bleeding in patients taking non-vitamin $\mathrm{K}$ antagonist oral anticoagulants or Warfarin. Am J Gastroenterol. 2017;112:734-739.

11. Reilly PA, Lehr T, Haertter S, et al. The effect of dabigatran plasma concentrations and patient characteristics on the frequency of ischemic stroke and major bleeding in atrial fibrillation patients: the RE-LY Trial (Randomized Evaluation of Long-Term Anticoagulation Therapy). J Am Coll Cardiol. 2014;63:321-328.

12. Abraham NS, Singh S, Alexander GC, et al. Comparative risk of gastrointestinal bleeding with dabigatran, rivaroxaban, and warfarin: population based cohort study. BMJ. 2015;350:h1857.

13. Cohen D. Dabigatran: how the drug company withheld important analyses. BMJ. 2014;349:g4670. 